\title{
Sicherung oder soziale Reintegration
}

\author{
H.-L. Kröber
}

Online publiziert: 8. Oktober 2011

(C) Springer-Verlag 2011

Das Urteil des Bundesverfassungsgerichts (BVerfG) vom 04.05.2011 hat die Regelungen zur Sicherungsverwahrung für grundrechtswidrig erklärt und dem Gesetzgeber die Neuregelung diverser Aspekte der Sicherungsverwahrung aufgegeben. Das Urteil ist für die forensische Psychiatrie und Psychologie mit dem Mangel behaftet, dass es das Therapieunterbringungsgesetz (ThUG) als unproblematischen Maßstab behandelt und keine kritischen Überlegungen zur Nutzung des Sachverhalts ,,psychische Störung“ als Begründung für unbefristete Freiheitsentziehung anstellt. Ansonsten aber hat das Urteil die positive Wirkung, dass die immer leichtere Anordnung und Ausweitung der Sicherungsverwahrung erst einmal gestoppt wird und dass die Eingrenzung auf schwere Straftaten unterstrichen wird. Faktisch verlangt das BVerfG, dass das Reintegrationsgebot nicht nur für Strafgefangene, sondern auch für Sicherungsverwahrte gelten muss. Die Entscheidung steht insofern in der Tradition der Entscheidung vom 21.06.1977 (BVerfGE 45, 187) zur lebenslangen Freiheitsstrafe. Damals hieß es: „Zu den Voraussetzungen eines menschenwürdigen Strafvollzugs gehört, dass dem zu lebenslanger Freiheitsstrafe Verurteilten grundsätzlich eine Chance verbleibt, je wieder der Freiheit teilhaftig zu werden. Die Möglichkeit der Begnadigung allein ist nicht ausreichend; vielmehr gebietet das Rechtsstaatsprinzip, die Voraussetzungen, unter denen die Vollstreckung einer lebenslangen Freiheitsstrafe ausgesetzt werden kann, und das dabei anzuwendende Verfahren gesetzlich zu regeln." Gleiches muss fraglos auch für die Sicherungsverwahrung gelten, und die Voraussetzungen, unter denen die

Prof. Dr. med. H.-L. Kröber $(\bowtie)$ Institut für Forensische Psychiatrie, Charité-Universitätsmedizin Berlin, Oranienburger Str. 285, 13437 Berlin, Deutschland E-Mail: Hans-Ludwig.Kroeber@charite.de
Maßregel ausgesetzt werden kann, sind mit der Angabe von Überprüfungsfristen nicht hinreichend beschrieben.

Entsprechend den Vorgaben des Verfassungsgerichts überlegen inzwischen das Bundesministerium der Justiz und die Länder, wie die konkrete Ausgestaltung der Sicherungsverwahrung aussehen muss, und man ahnt, dass es teuer wird. Insofern wäre es wichtig, die Überstellung in die Sicherungsverwahrung, wenn eben möglich, zu vermeiden, entweder durch Nichtanordnung oder durch eine Konzentration der Aktivitäten auf die Haftjahre, um den Vollzug der Maßregel überflüssig zu machen.

Im vorletzten Heft dieser Zeitschrift haben wir uns mit Vorschlägen zur Ausgestaltung der Sicherungsverwahrung befasst, im letzten Heft mit dem ThUG und der ,psychischen Störung", und in diesem Heft bleiben wir erneut bei dieser Problematik. Bernd Maelicke, der sein ganzes Berufsleben der Veränderung des Strafvollzugs in Richtung auf eine menschengerechte Sozialisierung gewidmet hat, beschreibt die Defizite im Umgang mit Mehrfach- und Intensivtätern und verdeutlicht in thesenartiger Verdichtung die weiterhin bestehenden Baustellen in der schrittweisen Reform des Strafvollzugs. Sodann verdeutlicht Andreas Mosbacher die juristischen Konsequenzen für den Umgang mit Sicherungsverwahrten, welche nun Entlassung begehren, und beschreibt insbesondere, wie der gutachterlich Tätige die Vorgabe des BVerfG verstehen könnte, eine „hochgradige Gefahr schwerster Gewalt- oder Sexualstraftaten aus konkreten Umständen in der Person oder dem Verhalten des Betroffenen abzuleiten“. Zugleich beschreibt er das juristische Verständnis des Terminus „psychische Störung“ im gegebenen Kontext. Der Unterzeichner hat, komplementär zu Mosbacher, zu begründen versucht, warum der Begriff „psychische Störung“" auch als Rechtsbegriff mit erfahrungswissenschaftlich beschreibbarem Inhalt gefüllt sein muss; dies führt zu der Folgerung, dass nur solche ,psychischen 
Störungen" künftige Gefährlichkeit verursachen, welche die Einsichtsfähigkeit aufheben oder aber das Hemmungsvermögen gegenüber rechtswidrigen Tatwünschen erheblich beeinträchtigen. Ist die Beeinträchtigung nicht erheblich, ist die psychische Störung für das Straftatrisiko logischerweise belanglos.

Der Beitrag von Michael Alex zeigt und diskutiert die Daten der Bochumer Studie zur Rückfälligkeit von Männern, gegen die nachträgliche Sicherungsverwahrung beantragt, aber nicht angeordnet worden war. Diese wesentlichen Ergebnisse gingen ja schon vor vielen Monaten durch die Presse; es wurden $60 \%$ dieser 77 Entlassenen bisher nicht erneut straffällig. Betrachtet man die Zusammensetzung der Gruppe, zeigt sich, dass natürlich keine Vergleichbarkeit mit den Altfällen der (primär angeordneten) Sicherungsverwahrung besteht. Vielmehr wurden nach Einführung der nachträglichen Sicherungsverwahrung von den Haftanstalten und dann den Staatsanwaltschaften sehr viele Gefangene für die nachträgliche Sicherungsverwahrung vorgeschlagen, nur weil sie die formalen Voraussetzungen erfüllten. Es wurden 21 der 77 nicht einmal begutachtet, weitere 10 von Gutachtern als wenig gefährlich eingestuft. Von den 32 Gefangenen, die gutachterlich als hochgefährlich einge- stuft wurden, wurden bislang 14 (44\%) wieder verurteilt, davon $10(31 \%)$ zu Freiheitsstrafen. Wir gehen also weiter davon aus, dass wir bei Hochgefährlichen ein längerfristiges Rückfallrisiko mit schweren Delikten von 40-50 \% haben. Das ist hoch, und keiner würde eine Heizanlage einbauen, die in den nächsten 5 Jahren mit 30-\%er Wahrscheinlichkeit explodiert. Zugleich rechtfertigt dieses Risiko die erheblichen Anstrengungen der ambulanten Nachsorge und Kontrolle, die nun für forciert entlassene Sicherungsverwahrte unternommen werden; Tatjana Voß et al. verdeutlichen dies an Fallbeispielen und beleuchten die Arbeitsweise der forensisch-therapeutischen Ambulanz. Das „Blitzlicht“ dieses Hefts korrespondiert damit in seinen Anmerkungen zum Übergangsmanagement.

In den freien Beiträgen widmet sich Peter Kalus der Rolle der bildgebenden Verfahren im heutigen Strafprozess. Thomas Görgen et al. geben eine ausführliche Übersicht über die Erkenntnisse in der wichtigen und heiklen Frage der Folgen sexuellen Missbrauchs für das betroffene Kind, und Benno Zabel befasst sich aus juristischer Sicht mit den rechtsdogmatischen Verwerfungen, die sich aus der Abschaffung des Straftatbestands der Kindstötung unter der Geburt ergeben haben. 